\title{
Bour's theorem and helicoidal surfaces with constant mean curvature in the Bianchi-Cartan-Vranceanu spaces
}

\author{
Renzo Caddeo ${ }^{1} \cdot$ Irene I. Onnis ${ }^{1} \cdot$ Paola Piu ${ }^{1}$
}

Received: 17 March 2021 / Accepted: 12 July 2021 / Published online: 11 August 2021

(c) The Author(s) 2021

\begin{abstract}
In this paper, we generalize a classical result of Bour concerning helicoidal surfaces in the three-dimensional Euclidean space $\mathbb{R}^{3}$ to the case of helicoidal surfaces in the BianchiCartan-Vranceanu (BCV) spaces, i.e., in the Riemannian 3-manifolds whose metrics have groups of isometries of dimension 4 or 6 , except the hyperbolic one. In particular, we prove that in a BCV-space there exists a two-parameter family of helicoidal surfaces isometric to a given helicoidal surface; then, by making use of this two-parameter representation, we characterize helicoidal surfaces which have constant mean curvature, including the minimal ones.
\end{abstract}

Keywords Helicoidal surfaces · Constant mean curvature surfaces $\cdot$ BCV spaces · Bour's theorem

Mathematics Subject Classification 53A10 - 53C40 - 53C42

\section{Introduction and preliminaries}

Helicoidal surfaces in the Euclidean three-dimensional space $\mathbb{R}^{3}$ are invariant under the action of the 1-parameter group of helicoidal motions and are a generalization of rotation surfaces. Since the beginning of differential geometry of surfaces, much attention has been given to the surfaces of revolution with constant Gauss curvature or constant mean curvature (CMC-surfaces). The surfaces of revolution with constant Gauss curvature seem to

Work supported by GNSAGA-INdAM, Italy, by Fondazione di Sardegna (Project STAGE) and Regione Autonoma della Sardegna (Project KASBA). The second author was also supported by the Thematic Project,: Topologia Álgebrica, Geométrica e Diferencial, Fapesp process number 2016/24707-4.

Paola Piu

piu@unica.it

Renzo Caddeo

caddeo@unica.it

Irene I. Onnis

irene.onnis@unica.it

1 Dipartimento di Matematica e Informatica, Università degli Studi di Cagliari, Via Ospedale 72, 09124 Cagliari, Italy 
have been known to Minding (1839, [24]), while those with constant mean curvature have been classified by Delaunay $(1841,[16])$. Helicoidal minimal surfaces were studied by Scherk in 1835 (see [37] and, also, [40]), but it is rather recent the classification of the helicoidal surfaces in $\mathbb{R}^{3}$ with nonzero constant mean curvature, given by Do Carmo and Dajczer in [18]. Also, in [35] Roussos proved that a helicoidal surface in $\mathbb{R}^{3}$ has constant mean curvature if and only if its principal axes make a constant angle with the orbits. Another important result in this direction was given independently in 1865 by Beltrami [3] and Dini [17] that classified ruled Weingarten surfaces (i.e., surfaces for which a non-trivial relation between their mean and Gauss curvatures holds) in $\mathbb{R}^{3}$, proving the following

Theorem 1 (Dini-Beltrami) Any non-developable ruled Weingarten surface in Euclidean 3-space $\mathbb{R}^{3}$ is a piece of a helicoidal ruled surface, defined as the orbit of a straight line under the action of a 1-parameter group of screw motions. In particular, the Gaussian curvature is nowhere zero if it is nonzero at some point. The only minimal ruled surface is the classical right helicoid.

Later, Kühnel presented in [22] a new and modern proof of this result. More recently, Dajczer and Tenenblat characterized the ruled Weingarten hypersurfaces in $\mathbb{R}^{n+1}, n \geq 3$, (see [14]).

These topics are related to the Bonnet problem ( [8]) which asks whether the knowledge of the metric and of the mean curvature is sufficient to determine a surface in $\mathbb{R}^{3}$; it has been treated not only from a classical viewpoint, but also from a modern viewpoint involving sophisticated techniques of integrable systems theory. In [12], Cartan proved that, beside the CMC surfaces in $\mathbb{R}^{3}$, the only ones admitting a non-trivial isometric deformation preserving the principal curvatures are finite-dimensional families of cones, cylinders or helicoidal immersions. In [34] Roussos showed that if a Bonnet surface with nonconstant mean curvature in the Euclidean 3-space is flat, then it is a piece of some deformation of the cylinder over a logarithmic spiral or a generalized cone. In [6], Bobenko and Eitner investigated the nontrivial Bonnet surfaces in $\mathbb{R}^{3}$ without umbilical points; a discussion of Bonnet surfaces with umbilics is contained in the dissertation of Eitner. In book [7], Bobenko and Eitner gave a detailed local and global description of Bonnet surfaces in $\mathbb{R}^{3}$ with and without umbilics. Also, the second half of the book is devoted to the study of Bonnet surfaces in $\mathbb{S}^{3}$ and $\mathbb{t}^{3}$. More recently, in [23] Jensen, Musso and Nicolodi obtained sufficient conditions for non-existence of compact Bonnet pairs in $\mathbb{R}^{3}$.

The starting point of the work [18] of do Carmo and Dajczer is a result of Bour about helicoidal surfaces in $\mathbb{R}^{3}$ (see [9], p. 82, Theorem II), for which he received the mathematics prize awarded by the Académie des Sciences de Paris in $1861^{1}$. Bour proved that there exists a 2-parameter family of helicoidal surfaces isometric to a given helicoidal surface in $\mathbb{R}^{3}$. For this, firstly he obtained orthogonal parameters $(u, t)$ on a helicoidal surface $M$ for which the families of $u$-coordinate curves are geodesics on $M$ parametrized by arc length,

\footnotetext{
1 The problem that sometimes bears the name of Bour was proposed in 1861 by the Académie des Sciences and consists in determining all the surfaces that are isometric to a given surface $\left(M, d s^{2}\right)$. E. Bour demonstrated that each helicoidal surface is applicable to a surface of revolution and that the helices on the first surface correspond to the parallels on the second. Bour's work [9] contains several theorems on ruled and minimal surfaces; but in its printed version this work does not include the complete integration of the problem's equations in the case of surfaces of revolution; in fact, it is this result that enabled Bour to win the Academy's grand prix.
} 
and the $t$-coordinate curves are the trajectories of the helicoidal motion. Such parameters are called natural parameters, and the first fundamental form with respect to them takes the form $d s^{2}=d u^{2}+U^{2}(u) d t^{2}$. Reciprocally, given the natural parameters $(u, t)$ on $M$ and a function $U(u)$, Bour determined a 2-parameter family of isometric helicoidal surfaces which have induced metric given by $d s^{2}=d u^{2}+U^{2}(u) d t^{2}$ that includes rotation surfaces. An exposition of Bour's results about the theory of deformation of surfaces can be found in the Chapter IX of [15].

By using the result of Bour, in [18] Do Carmo and Dajczer established a condition for a surface of the Bour's family to have constant mean curvature. Also they obtained an integral representation (depending on three parameters) of helicoidal surfaces with nonzero constant mean curvature, which is a natural generalization of the representation for Delaunay surfaces, i.e., CMC rotation surfaces, given by Kenmotsu (see [21]).

In [36], the authors obtain a generalized Bour's theorem for helicoidal surfaces in the products $\mathbb{H}^{2} \times \mathbb{R}$ and $\mathbb{S}^{2} \times \mathbb{R}$ and use it to determine all isometric immersions in these spaces that give the surfaces which are helicoidal and have the same constant mean curvature.

In regard to the study of CMC helicoidal surfaces in BCV spaces, in [19] and in [25, 29] the authors use the equivariant geometry to classify the profile curves of these surfaces in the Heisenberg group $\mathbb{H}_{3}$ and in $\mathbb{\boxplus}^{2} \times \mathbb{R}$, respectively. The case of rotational minimal and constant mean curvature surfaces in the Heisenberg group is treated in [10]. J. Ripoll in $[32,33]$ classified the CMC invariant surfaces in the 3 -dimensional sphere $\mathbb{S}^{3}$ and also in the hyperbolic 3-space $\mathbb{H}^{3}$.

The aim of this paper is to generalize the results obtained in [18] and [36]. The paper is organized as follows. Section 2 is devoted to give a short description of the Bianchi-Cartan-Vranceanu spaces and the helicoidal surfaces in these spaces. In Sect. 3, we establish a Bour's type theorem for helicoidal surfaces in the BCV spaces (see Theorem 2) and, as an immediate consequence of this result, we have that every helicoidal surface in a BCVspace can be isometrically deformed into a rotation surface through helicoidal surfaces. Moreover, Corollary 1 refers to the particular case of isometric rotation surfaces.

In Sect. 4, we use techniques of equivariant geometry, in particular the Reduction Theorem of Back, do Carmo and Hsiang (see [2]), to deduce a differential equation that the function $U(u)$ must satisfy in order that a helicoidal surface of the Bour's family determined by $U(u)$ has constant mean curvature. We solve this equation by making a transformation of coordinates, treating separately the case of the space forms $\mathbb{R}^{3}$ and $\mathbb{S}^{3}$ from the other BCV spaces. In this way, we obtain Theorem 4 that provides a description, in terms of natural parameters, of all helicoidal surfaces of constant mean curvature in a BCVspace, including the minimal ones. We conclude by showing that in $\mathbb{R}^{3}$ these results give a natural parametrization of all the helicoidal minimal surfaces obtained by Scherk in [37].

\section{Helicoidal surfaces in Bianchi-Cartan-Vranceanu spaces}

A Riemannian manifold $(\mathcal{M}, g)$ is said to be homogeneous if for every two points $p$ and $q$ in $\mathcal{M}$, there exists an isometry of $\mathcal{M}$, mapping $p$ into $q$. The classification of 3-dimensional simply connected homogeneous spaces is well known and can be summarized as follows. First of all, the dimension of the isometry group must be equal to 6, 4 or 3 (see [4] or [20]). Then, if the isometry group is of dimension $6, \mathcal{M}$ is a complete real space form, i.e., the Euclidean space $\mathbb{E}^{3}$, a sphere $\mathbb{S}^{3}(k)$, or a hyperbolic space $\mathbb{H}^{3}(k)$. If the dimension of the 
isometry group is $4, \mathcal{M}$ is isometric to $\mathrm{SU}(2)$, the special unitary group, to $\widetilde{\operatorname{SL}(2, R)}$, the universal covering of the real special linear group, to $\mathrm{Nil}_{3}$, the Heisenberg group, all with a certain left-invariant metric, or to a Riemannian product $\mathbb{S}^{2}(k) \times \mathbb{R}$ or $\mathbb{M}^{2}(k) \times \mathbb{R}$. Finally, if the dimension of the isometry group is $3, \mathcal{M}$ is also isometric to a simply connected Lie group with a left-invariant metric, for example, that is called SOL, one of the Thurston's eight models of geometry [38].

An explicit classification of 3-dimensional homogeneous Riemannian metrics based on the dimension of their isometry group was first given by Luigi Bianchi in 1897 (see [4] or [5]). Later Élie Cartan in [11] and Gheorghe Vranceanu in [39] proved that all the metrics whose group of isometries has dimension 4 or 6 , except the hyperbolic one, can be represented in a concise form by the following two-parameter family of metrics

$$
g_{\kappa, \tau}=\frac{d x^{2}+d y^{2}}{B^{2}}+\left(d z+\tau \frac{y d x-x d y}{B}\right)^{2},
$$

for $\kappa, \tau \in \mathbb{R}$, and $B=1+\frac{\kappa}{4}\left(x^{2}+y^{2}\right),(x, y, z) \in \mathbb{R}^{3}$, positive. Thus, the family of metrics $g_{\kappa, \tau}$ that can rightfully be named the Bianchi-Cartan-Vranceanu metrics (BCV metrics) consists of all three-dimensional homogeneous metrics whose group of isometries has dimension 4 or 6 , except for those of constant negative sectional curvature. In the following, we shall denote by $\mathcal{N}_{\kappa, \tau}$ the open subset of $\mathbb{R}^{3}$ where the metrics $g_{\kappa, \tau}$ are defined.

With respect to (1), we have the following globally defined orthonormal frame

$$
E_{1}=B \frac{\partial}{\partial x}-\tau y \frac{\partial}{\partial z}, \quad E_{2}=B \frac{\partial}{\partial y}+\tau x \frac{\partial}{\partial z}, \quad E_{3}=\frac{\partial}{\partial z}
$$

and, also,

Proposition 1 ([30,31]) The isometry group of $g_{\kappa, \tau}$ admits the basis of Killing vector fields

$$
\left\{\begin{array}{l}
X_{1}=\left(1-\frac{\kappa y^{2}}{2 B}\right) E_{1}+\frac{\kappa x y}{2 B} E_{2}+\frac{2 \tau y}{B} E_{3}, \\
X_{2}=\frac{\kappa x y}{2 B} E_{1}+\left(1-\frac{\kappa x^{2}}{2 B}\right) E_{2}-\frac{2 \tau x}{B} E_{3}, \\
X_{3}=-\frac{y}{B} E_{1}+\frac{x}{B} E_{2}-\frac{\tau\left(x^{2}+y^{2}\right)}{B} E_{3}, \\
X_{4}=E_{3} .
\end{array}\right.
$$

Therefore, the group of isometries of the BCV spaces contains the helicoidal subgroup, whose infinitesimal generator is the Killing vector field given by

$$
X=-y \frac{\partial}{\partial x}+x \frac{\partial}{\partial y}+a \frac{\partial}{\partial z}, \quad a \in \mathbb{R} .
$$

We consider the surfaces in $\mathcal{N}_{\kappa, \tau}$ which are invariant under the action of the one-parameter group of isometries $G_{X}$ of $g_{\kappa, \tau}$ generated by $X$. For convenience, we shall introduce cylindrical coordinates 


$$
\left\{\begin{array}{l}
x=r \cos \theta \\
y=r \sin \theta \\
z=z
\end{array}\right.
$$

with $r \geq 0$ and $\theta \in(0,2 \pi)$. In these coordinates, the metric (1) becomes

$$
g_{\kappa, \tau}=\frac{d r^{2}}{B^{2}}+r^{2}\left(\frac{1+\tau^{2} r^{2}}{B^{2}}\right) d \theta^{2}+d z^{2}-2 \frac{\tau r^{2}}{B} d \theta d z
$$

where $B=1+\frac{\kappa}{4} r^{2}$. Moreover, the Killing vector field $X$ takes the form

$$
X=\frac{\partial}{\partial \theta}+a \frac{\partial}{\partial z}
$$

and a set of two invariant functions is

$$
\xi_{1}=r, \quad \xi_{2}=z-a \theta .
$$

Thus, the orbit space of the action of $G_{X}$ can be identified with

$$
\mathcal{B}:=\mathcal{N}_{\kappa, \tau} / G_{X}=\left\{\left(\xi_{1}, \xi_{2}\right) \in \mathbb{R}^{2}: \xi_{1} \geq 0\right\}
$$

and the orbital distance metric of $\mathcal{B}$ is given by

$$
\widetilde{g}=\frac{d \xi_{1}^{2}}{B^{2}}+\frac{\xi_{1}^{2} d \xi_{2}^{2}}{\xi_{1}^{2}+\left(a B-\tau \xi_{1}^{2}\right)^{2}}
$$

where $B=1+\frac{\kappa}{4} \xi_{1}^{2}$.

Now, consider a helicoidal surface $M$ (with pitch $a$ ) that, locally, with respect to the cylindrical coordinates, can be parametrized by

$$
\psi(u, \theta)=\left(\xi_{1}(u), \theta, \xi_{2}(u)+a \theta\right),
$$

and suppose that the profile curve $\tilde{\gamma}(u)=\left(\xi_{1}(u), \xi_{2}(u)\right)$ is parametrized by arc-length in $(\mathcal{B}, \widetilde{g})$, so that

$$
\frac{\xi_{1}^{\prime 2}}{B^{2}}+\frac{\xi_{1}^{2} \xi_{2}^{\prime 2}}{\xi_{1}^{2}+\left(a B-\tau \xi_{1}^{2}\right)^{2}}=1
$$

Therefore, from

$$
\left\{\begin{array}{l}
\psi_{u}=\xi_{1}^{\prime}\left(\frac{\cos \theta}{B} E_{1}+\frac{\sin \theta}{B} E_{2}\right)+\xi_{2}^{\prime} E_{3}, \\
\psi_{\theta}=\frac{\xi_{1}}{B}\left(\cos \theta E_{2}-\sin \theta E_{1}\right)+\left(a-\frac{\tau \xi_{1}^{2}}{B}\right) E_{3}=X
\end{array}\right.
$$

it follows that the coefficients of the induced metric of the helicoidal surface are given by

$$
E(u)=1+\xi_{2}^{\prime}(u)^{2}\left(\frac{a B(u)-\tau \xi_{1}(u)^{2}}{B(u) \omega(u)}\right)^{2}, \quad F(u)=\xi_{2}^{\prime}(u)\left(\frac{a B(u)-\tau \xi_{1}(u)^{2}}{B(u)}\right),
$$

and 


$$
G=\frac{\xi_{1}(u)^{2}}{B(u)^{2}}+\left(a-\frac{\tau \xi_{1}(u)^{2}}{B(u)}\right)^{2}=\omega(u)^{2},
$$

where $\omega(u)$ is the volume function of the principal orbit.

\section{A Bour's type theorem}

In this section, we show that every helicoidal surface in a BCV-space admits a reparametrization by natural parameters and, conversely, given a positive function $U$, it is possible to find a 2-parameter family of isometric helicoidal surfaces associated with it that are parameterized by natural parameters.

Theorem 2 In the BCV-space $\mathcal{N}_{\kappa, \tau}$ there exists a two-parameter family of helicoidal surfaces that are isometric to a given helicoidal surface of the form (6) and that includes a rotation surface. More precisely, for a given positive function $U(u)$ and arbitrary constants $m \neq 0$ and $a$, the helicoidal surfaces (6) whose profile curve $\tilde{\gamma}(u)=\left(\xi_{1}(u), \xi_{2}(u)\right)$ is given by

$$
\left\{\begin{array}{l}
\xi_{1}(u)=2 \sqrt{\frac{m^{2} U^{2}-a^{2}}{(1+\sqrt{\Delta})^{2}-4 \tau^{2} m^{2} U^{2}}}, \\
\xi_{2}(u)=\int \frac{m U\left(4+\kappa \xi_{1}^{2}\right)}{4 \xi_{1}^{2}} \sqrt{\xi_{1}^{2}-\frac{m^{4} U^{2} U^{\prime 2}\left(4+\kappa \xi_{1}^{2}\right)^{2}}{16 \Delta}} d u,
\end{array}\right.
$$

with

$$
\theta(u, t)=\frac{t}{m}+\int \frac{(4 \tau-a \kappa) \xi_{1}^{2}-4 a}{4 m U \xi_{1}^{2}} \sqrt{\xi_{1}^{2}-\frac{m^{4} U^{2} U^{\prime 2}\left(4+\kappa \xi_{1}^{2}\right)^{2}}{16 \Delta}} d u
$$

where

$$
\Delta(u)=(1-2 a \tau)^{2}+\left(m^{2} U(u)^{2}-a^{2}\right)\left(4 \tau^{2}-\kappa\right),
$$

are all to each other isometric and have first fundamental form given by $d u^{2}+U(u)^{2} d t^{2}$.

Proof From (8), we have that the induced metric of a helicoidal surface (6), with pitch $a_{0}$, is given by

$$
\begin{aligned}
g_{\psi} & =E(u) d u^{2}+2 F(u) d u d \theta+\omega(u)^{2} d \theta^{2} \\
& =d u^{2}+\omega(u)^{2}\left(d \theta+\xi_{2}^{\prime}(u) \frac{a_{0} B(u)-\tau \xi_{1}(u)^{2}}{B(u) \omega(u)^{2}} d u\right)^{2},
\end{aligned}
$$

where

$$
\omega(u)^{2}=\frac{\xi_{1}(u)^{2}}{B(u)^{2}}+\left(\frac{a_{0} B(u)-\tau \xi_{1}(u)^{2}}{B(u)}\right)^{2} .
$$


Now we introduce a new parameter $t=t(u, \theta)$ that satisfies:

$$
d t=d \theta+\xi_{2}^{\prime}(u) \frac{a_{0} B(u)-\tau \xi_{1}(u)^{2}}{B(u) \omega(u)^{2}} d u .
$$

As the Jacobian $|\partial(u, t) / \partial(u, \theta)|$ is equal to 1 , it follows that $(u, t)$ are local coordinates on a helicoidal surface $M$ and, also, that we can write (11) as

$$
g_{\psi}=d u^{2}+\omega(u)^{2} d t^{2} .
$$

We now observe that the $u$-coordinate curves are parametrized by arc length and also that are geodesics of $M$ (see [28]) which are orthogonal to the $t$-coordinate curves, i.e., the helices. Consequently, the local parametrization $\psi(u, \theta(u, t))$ is a natural parametrization of the helicoidal surface $M$.

Conversely, given a function $U(u)>0$, we want to determine functions $\theta, \xi_{1}, \xi_{2}$ of $(u, t)$ such that

$$
\left\{\begin{aligned}
d u^{2} & =\frac{d \xi_{1}^{2}}{B^{2}}+\frac{\xi_{1}^{2} d \xi_{2}^{2}}{\xi_{1}^{2}+\left(a B-\tau \xi_{1}^{2}\right)^{2}} \\
\pm U(u) d t & =\frac{\sqrt{\xi_{1}^{2}+\left(a B-\tau \xi_{1}^{2}\right)^{2}}}{B}\left[d \theta+\frac{B\left(a B-\tau \xi_{1}^{2}\right)}{\xi_{1}^{2}+\left(a B-\tau \xi_{1}^{2}\right)^{2}} d \xi_{2}\right]
\end{aligned}\right.
$$

where $B=1+\frac{\kappa}{4} \xi_{1}^{2}$.

From the first equation of (14), we have that $\xi_{i}=\xi_{i}(u), i=1,2$. Then, from the second, we obtain

$$
\left\{\begin{array}{l}
\frac{\partial \theta}{\partial u}=-\frac{B(u)\left[a B(u)-\tau \xi_{1}^{2}(u)\right]}{\xi_{1}^{2}(u)+\left(a B(u)-\tau \xi_{1}^{2}(u)\right)^{2}} \xi_{2}^{\prime}(u), \\
\frac{\partial \theta}{\partial t}= \pm \frac{B(u) U(u)}{\sqrt{\xi_{1}^{2}(u)+\left(a B(u)-\tau \xi_{1}^{2}(u)\right)^{2}}},
\end{array}\right.
$$

where $B(u)=1+\frac{\kappa}{4} \xi_{1}^{2}(u)$.

Therefore,

$$
\frac{\partial^{2} \theta}{\partial t \partial u}=0
$$

and hence, there exists a constant $m \neq 0$ such that

$$
\pm \frac{B(u) U(u)}{\sqrt{\xi_{1}^{2}(u)+\left(a B(u)-\tau \xi_{1}^{2}(u)\right)^{2}}}=\frac{1}{m} .
$$

Thus, the second equation of system (14) becomes 


$$
d \theta=\frac{d t}{m}-\frac{B(u)\left(a B(u)-\tau \xi_{1}^{2}(u)\right)}{\xi_{1}^{2}(u)+\left(a B(u)-\tau \xi_{1}^{2}(u)\right)^{2}} d \xi_{2} .
$$

If we consider the function $f:=1 / \xi_{1}$, equation (16) can be written as

$$
\left(a^{2}-m^{2} U^{2}\right)\|\nabla f\|_{\kappa, \tau}^{2}+(1-2 a \tau)\|\nabla f\|_{\kappa, \tau}+\tau^{2}-\kappa / 4=0
$$

and therefore,

$$
\|\nabla f\|_{\kappa, \tau}=\frac{1-2 a \tau+\sqrt{\Delta}}{2\left(m^{2} U^{2}-a^{2}\right)},
$$

with

$$
\Delta=(1-2 a \tau)^{2}+\left(m^{2} U^{2}-a^{2}\right)\left(4 \tau^{2}-\kappa\right) .
$$

As $\|\nabla f\|_{\kappa, \tau}=f^{2}+\kappa / 4$, we conclude that

$$
\xi_{1}^{2}=\frac{4\left(m^{2} U^{2}-a^{2}\right)}{(1+\sqrt{\Delta})^{2}-4 \tau^{2} m^{2} U^{2}} .
$$

Then, differentiating (16) and using (18), we get

$$
m^{2} B^{2} U U^{\prime}=\sqrt{\Delta} \xi_{1} \xi_{1}^{\prime}
$$

and hence,

$$
\frac{\left(\xi_{1}^{\prime}\right)^{2}}{B^{2}}=\frac{m^{4} B^{2} U^{2} U^{\prime 2}}{\xi_{1}^{2} \Delta} .
$$

Therefore, taking into account the first equation of system (14), we obtain

$$
\begin{aligned}
d \xi_{2}^{2} & =\frac{m^{2} B^{2} U^{2}}{\xi_{1}^{2}}\left(1-\frac{\xi_{1}^{\prime 2}}{B^{2}}\right) d u^{2} \\
& =\frac{m^{2} B^{2} U^{2}}{\xi_{1}^{4}}\left(\xi_{1}^{2}-\frac{m^{4} B^{2} U^{2} U^{\prime 2}}{\Delta}\right) d u^{2} .
\end{aligned}
$$

Thus, as

$$
B=1+\frac{\kappa}{4} \xi_{1}^{2}
$$

it turns out that

$$
\xi_{2}(u)=\int \frac{m U\left(4+\kappa \xi_{1}^{2}\right)}{4 \xi_{1}^{2}} \sqrt{\xi_{1}^{2}-\frac{m^{4} U^{2} U^{\prime 2}\left(4+\kappa \xi_{1}^{2}\right)^{2}}{16 \Delta}} d u .
$$

Also, from (17) we have 


$$
\theta(u, t)=\frac{t}{m}+\int \frac{(4 \tau-a \kappa) \xi_{1}^{2}-4 a}{4 m U \xi_{1}^{2}} \sqrt{\xi_{1}^{2}-\frac{m^{4} U^{2} U^{\prime 2}\left(4+\kappa \xi_{1}^{2}\right)^{2}}{16 \Delta}} d u .
$$

Consequently, the natural parametrization of the helicoidal surface (6) with given first fundamental form $g_{\psi}=d u^{2}+U(u)^{2} d t^{2}$ can be calculated by means of equations (18), (21) and (22).

Remark 1 If $\kappa=0=\tau$, the BCV-space is the Euclidean space $\mathbb{R}^{3}$ and Theorem 2 becomes the classical one ([9], p. 82, Theorem II) due to Bour.

Remark 2 The family of helicoidal surfaces $\Psi(u, t):=\psi_{[U, m, a]}(u, t)$ in the BCV-space $\mathcal{N}_{\kappa, \tau}$ obtained in Theorem 2 depends on two parameters $m \neq 0$ and $a$, and for $m=1$ and $a=a_{0}$ it contains the original helicoidal surface. Also, when $m=1$ and $a=0$, we obtain a rotational surface isometric to the given helicoidal surface. Therefore, by varying the constant $a$ from $a=0$ to $a=a_{0}$, we get an isometric deformation from a rotational surface to a given helicoidal surface.

Example 1 In the Heisenberg space $\mathbb{H}_{3}$ equipped with the metric $g_{\kappa, \tau}$ with $\kappa=0$ and $\tau=1 / 2$, we consider the function $U(u)=\left(u^{2}+2\right) / 2$. If we suppose that $m=1$, from formulas (9) we get

$$
\left\{\begin{array}{l}
\xi_{1}(u)=\sqrt{\sqrt{u^{4}+4 u^{2}+8(1-a)}+2(a-1)}, \\
\xi_{2}(u)=\int \frac{\left(2+u^{2}\right)}{2 \xi_{1}(u)^{2}} \sqrt{\xi_{1}(u)^{2}-\frac{u^{2}\left(u^{2}+2\right)^{2}}{u^{4}+4 u^{2}+8(1-a)}} d u, \\
\theta(u, t)=t+\int \frac{\xi_{1}(u)^{2}-2 a}{\left(u^{2}+2\right) \xi_{1}(u)^{2}} \sqrt{\xi_{1}(u)^{2}-\frac{u^{2}\left(u^{2}+2\right)^{2}}{u^{4}+4 u^{2}+8(1-a)}} d u .
\end{array}\right.
$$

In particular, for $a=1 / 2$ we obtain the curve

$$
\tilde{\gamma}(u)=\left(\sqrt{u^{2}+1},(u+\arctan u) / 2\right),
$$

the profile curve of the helicoidal catenoid that is a helicoidal minimal surface (see [19]), parametrized by

$$
\psi(u, \theta)=\left(\sqrt{u^{2}+1} \cos \theta, \sqrt{u^{2}+1} \sin \theta, \frac{u+\theta+\arctan u}{2}\right) .
$$

Also, as

$$
\theta(u, t)=t-\arctan u+\sqrt{2} \arctan \left(\frac{u}{\sqrt{2}}\right),
$$


Fig. 1 Isometric deformation of the helicodal catenoid into a rotation surface in $\mathbb{H}_{3}$. The surfaces in the picture are obtained for $a=1 / 2,1 / 4,1 / 8$ and $a=0$, respectively; only that with angular pitch $a=1 / 2$ is a minimal surface (see Remark 5)

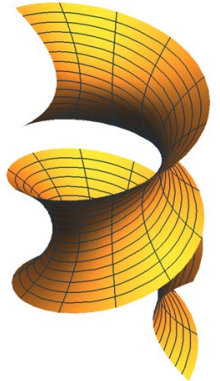

$a=1 / 2$

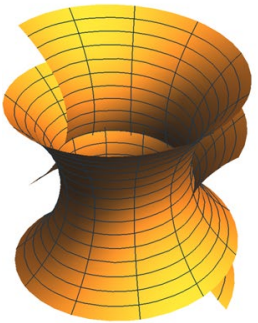

$a=1 / 8$

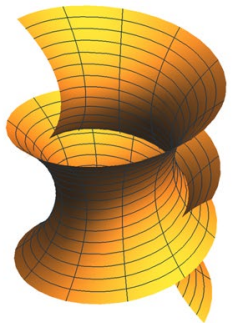

$a=1 / 4$

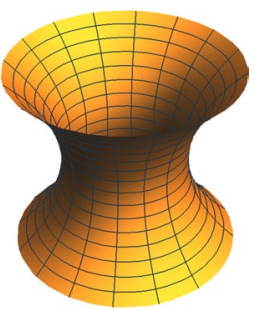

$a=0$

we have that the parametrization

$$
\begin{aligned}
\Psi(u, t) & =\psi(u, \theta(u, t))=\left(\cos \left(t+\sqrt{2} \arctan \left(\frac{u}{\sqrt{2}}\right)\right)+u \sin \left(t+\sqrt{2} \arctan \left(\frac{u}{\sqrt{2}}\right)\right),\right. \\
& \left.\sin \left(t+\sqrt{2} \arctan \left(\frac{u}{\sqrt{2}}\right)\right)-u \sin \left(t+\sqrt{2} \arctan \left(\frac{u}{\sqrt{2}}\right)\right), \frac{1}{2}\left(u+t+\sqrt{2} \arctan \left(\frac{u}{\sqrt{2}}\right)\right)\right)
\end{aligned}
$$

represents a natural parametrization of the helicoidal catenoid with

$$
g_{\Psi}=d u^{2}+U(u)^{2} d t^{2} .
$$

Now, if we start from $a=1 / 2$ and in the equations (23) we consider all the decreasing values of $a$ in the interval $[0,1 / 2]$, we obtain an isometric deformation of the helicoidal catenoid into a rotational surface (obtained for $a=0$ ), through helicoidal surfaces parametrized by natural parameters Fig. 1 .

Corollary 1 The rotation surfaces given by $\psi_{[U, n, 0]}(u, t)$, with $n \neq 0$, give rise in the $B C V$ space $\mathcal{N}_{\kappa, \tau}$ to a 1-parameter family of isometric surfaces that are also isometric to the helicoidal surfaces $\psi_{[U, m, a]}(u, t)$. This family is determined by the formulas: 


$$
\left\{\begin{array}{l}
\xi_{1}(u)=\frac{2 n U}{\sqrt{2(1+\sqrt{\Delta})-\kappa n^{2} U^{2}}}, \\
\xi_{2}(u)=\int \sqrt{\frac{(1+\sqrt{\Delta})^{2}}{2(1+\sqrt{\Delta})-\kappa n^{2} U^{2}}-\frac{n^{2}(1+\sqrt{\Delta})^{4} U^{2}}{\Delta\left[2(1+\sqrt{\Delta})-\kappa n^{2} U^{2}\right]^{2}}} d u, \\
\theta(u, t)=\frac{t}{n}+2 \tau \int \sqrt{\frac{1}{2(1+\sqrt{\Delta})-\kappa n^{2} U^{2}}-\frac{n^{2}(1+\sqrt{\Delta})^{2} U^{\prime 2}}{\Delta\left[2(1+\sqrt{\Delta})-\kappa n^{2} U^{2}\right]^{2}}} d u,
\end{array}\right.
$$

where $\Delta=1+\left(4 \tau^{2}-\kappa\right) n^{2} U^{2}$.

From the formula for the Gaussian curvature of an invariant surface obtained in [26, 27], it follows that the helicoidal surfaces of the Bour's family in the BCV-space $\mathcal{N}_{\kappa, \tau}$ have all the same Gaussian curvature given by

$$
K(u)=-\frac{U^{\prime \prime}(u)}{U(u)} .
$$

With regard to the mean curvature $H$ of these surfaces, in next section we shall see that different values of $a$ and $m$ can give rise to different values of $H$.

\section{Helicoidal surfaces of constant mean curvature}

In this section, we will describe the helicoidal surfaces in the BCV spaces that have the same constant mean curvature. We start by computing the mean curvature of a helicoidal surface (6). It turns out that the mean curvature of an invariant immersion is tightly related to the geodesic curvature of the profile curve, as shown by the remarkable following theorem. But first we recall that if on a three-dimensional connected Riemannian manifold $\left(N^{3}, g\right)$, we consider the 1-parameter subgroup $G_{X}$ of isometries generated by $X$, an orbit $G(p)$ of $p \in N$ is called principal if there exists an open neighborhood $U \subset N$ of $p$ such that all orbits $G(q), q \in U$, are of the same type as $G(p)$ (i.e., the isotropy subgroups $G_{q}$ and $G_{p}$ are conjugated). This implies that $G(q)$ is diffeomorphic to $G(p)$. We denote with $N_{r}$ the regular part of $N$, that is, the subset consisting of points belonging to principal orbits [1]. Then, we have

Theorem 3 (Reduction Theorem [2]) Let $H$ be the mean curvature of a $G_{X^{-}}$-invariant surface $M_{r} \subset N_{r}$ and $k_{g}$ the geodesic curvature of the profile curve $M_{r} / G_{X} \subset \mathcal{B}_{r}$. Then,

$$
H(x)=k_{g}(\pi(x))-D_{\mathbf{n}} \ln \omega(\pi(x)), \quad x \in M_{r},
$$

where $\mathbf{n}$ is the unit normal of the profile curve and $\omega=\sqrt{g(X, X)}$ is the volume function of the principal orbit.

Let now $\tilde{\gamma}(u)=\left(\xi_{1}(u), \xi_{2}(u)\right)$ be a curve in $\mathcal{B}_{r}$, parametrized by arc-length, that under the action of $G_{X}$ generates the helicoidal surface. From (5), it follows that 


$$
\xi_{1}^{\prime}=B \cos \sigma, \quad \xi_{2}^{\prime}=\frac{\sqrt{\xi_{1}^{2}+\left(a B-\tau \xi_{1}^{2}\right)^{2}} \sin \sigma}{\xi_{1}}
$$

and the geodesic curvature of $\tilde{\gamma}$ takes the expression

$$
\begin{aligned}
k_{g}= & \frac{\left(\widetilde{g}_{22}\right)_{\xi_{1}} \xi_{2}^{\prime}-\left(\widetilde{g}_{11}\right)_{\xi_{2}} \xi_{1}^{\prime}}{2 \sqrt{\widetilde{g}_{11} \widetilde{g}_{22}}}+\sigma^{\prime} \\
& =\frac{B\left[\xi_{1}^{2}+\left(a B-\tau \xi_{1}^{2}\right)^{2}\right]\left(\widetilde{g}_{22}\right)_{\xi_{1}}}{2 \xi_{1}^{2}} \sin \sigma+\sigma^{\prime},
\end{aligned}
$$

where $\sigma$ is the angle that $\tilde{\gamma}$ makes with the $\frac{\partial}{\partial \xi_{1}}$ direction. Also, as

$$
\mathbf{n}=\left(-B \sin \sigma, \frac{\sqrt{\xi_{1}^{2}+\left(a B-\tau \xi_{1}^{2}\right)^{2}}}{\xi_{1}} \cos \sigma\right),
$$

the normal derivative is given by

$$
D_{\mathbf{n}} \ln \omega=\frac{4 \xi_{1}\left[8 a \tau-4+\xi_{1}^{2}\left(\kappa+2 a \kappa \tau-8 \tau^{2}\right)\right] \sin \sigma}{16 \xi_{1}^{2}+\left[a\left(4+\kappa \xi_{1}^{2}\right)-4 \tau \xi_{1}^{2}\right]^{2}}
$$

and thus, we obtain that the mean curvature is given by

$$
H=\sigma^{\prime}+\left(\frac{1}{\xi_{1}}-\frac{\kappa}{4} \xi_{1}\right) \sin \sigma .
$$

Proposition 2 A helicoidal surface $\Psi(u, t):=\psi_{[U, m, a]}(u, t)$ has constant mean curvature $H$ if and only if $U(u)$ satisfies the differential equation

$$
H \sqrt{\frac{4\left(m^{2} U^{2}-a^{2}\right)}{(1+\sqrt{\Delta})^{2}-4 \tau^{2} m^{2} U^{2}}-\frac{m^{4} B^{2} U^{2} U^{\prime 2}}{\Delta}}=2-B-m^{2} B\left(\frac{U U^{\prime}}{\sqrt{\Delta}}\right)^{\prime},
$$

where

$$
\Delta=(1-2 a \tau)^{2}+\left(m^{2} U^{2}-a^{2}\right)\left(4 \tau^{2}-\kappa\right) \quad \text { and } \quad B=2 \frac{1-2 a \tau+\sqrt{\Delta}}{(1+\sqrt{\Delta})^{2}-4 \tau^{2} m^{2} U^{2}} .
$$

Proof If we consider a helicoidal surface $\Psi(u, t)$ of the Bour's family, from (25) we can write equation (27) as

$$
H=-\frac{\left(\frac{\xi_{1}^{\prime}}{B}\right)^{\prime}}{\sqrt{1-\left(\frac{\xi_{1}^{\prime}}{B}\right)^{2}}}+\left(\frac{1}{\xi_{1}}-\frac{\kappa}{4} \xi_{1}\right) \sqrt{1-\left(\frac{\xi_{1}^{\prime}}{B}\right)^{2}}
$$

and therefore, using (20) we get 


$$
\begin{aligned}
& -\left(\frac{\xi_{1}^{\prime}}{B}\right)^{\prime}+\left(\frac{1}{\xi_{1}}-\frac{\kappa}{4} \xi_{1}\right)\left[1-\left(\frac{\xi_{1}^{\prime}}{B}\right)^{2}\right]=H \sqrt{1-\left(\frac{\xi_{1}^{\prime}}{B}\right)^{2}} \\
& =\frac{H}{\xi_{1}} \sqrt{\xi_{1}^{2}-\frac{m^{4} B^{2} U^{2} U^{\prime 2}}{\Delta}} .
\end{aligned}
$$

Then,

$$
\begin{aligned}
& H \sqrt{\xi_{1}^{2}-\frac{m^{4} B^{2} U^{2} U^{\prime 2}}{\Delta}} \\
& =\xi_{1}\left[-\left(\frac{\xi_{1}^{\prime}}{B}\right)^{\prime}+\left(\frac{1}{\xi_{1}}-\frac{\kappa}{4} \xi_{1}\right)\left(1-\left(\frac{\xi_{1}^{\prime}}{B}\right)^{2}\right)\right] \\
& =1-\left(\frac{\xi_{1}^{\prime}}{B}\right)^{2}-\frac{\xi_{1} \xi_{1}^{\prime \prime}}{B}+\frac{3 \kappa \xi_{1}^{2}}{4}\left(\frac{\xi_{1}^{\prime}}{B}\right)^{2}-\frac{k}{4} \xi_{1}^{2} .
\end{aligned}
$$

Now, differentiating (19) we get

$$
\xi_{1} \xi_{1}^{\prime \prime}=\frac{m^{2} B^{2}}{\sqrt{\Delta}}\left(U^{\prime 2}+U U^{\prime \prime}\right)+\frac{m^{4} B^{2}}{\Delta}\left(\kappa B+\frac{\kappa-4 \tau^{2}}{\sqrt{\Delta}}\right) U^{2} U^{\prime 2}-\xi_{1}^{\prime 2}
$$

and hence, taking into account (18), (19) and (20), we can write equation (31) as

$$
\begin{aligned}
& H \sqrt{\xi_{1}^{2}-\frac{m^{4} B^{2} U^{2} U^{\prime 2}}{\Delta}} \\
& =2-B-\frac{m^{2} B}{\sqrt{\Delta}}\left(U^{\prime 2}+U U^{\prime \prime}\right)+\frac{m^{4} B}{\Delta}\left(\frac{4 \tau^{2}-\kappa}{\sqrt{\Delta}}-\frac{\kappa B}{4}+\frac{B^{2}-B}{\xi_{1}^{2}}\right) U^{2} U^{\prime 2} \\
& =2-B-\frac{m^{2} B}{\sqrt{\Delta}}\left(U^{\prime 2}+U U^{\prime \prime}\right)+\frac{\left(4 \tau^{2}-\kappa\right) m^{4} B}{\Delta^{3 / 2}} U^{2} U^{\prime 2}
\end{aligned}
$$

\subsection{The solution of the mean curvature equation}

Next, we will give a description of the helicoidal surfaces in $\mathcal{N}_{\kappa, \tau}$ with constant mean curvature $H$. For this purpose, we assume that the helicoidal surfaces are parametrized by natural coordinates $(u, t)$ and we determine explicitly the expression of the function $U(u)$ that gives the metric, by integrating (28).

Theorem 4 In the BCV-space $\mathcal{N}_{\kappa, \tau}, \quad$ the helicoidal surface $\Psi(u, t):=\left(\xi_{1}(u), \theta(u, t), \xi_{2}(u)+a \theta(u, t)\right)$ with $\xi_{1}(u), \xi_{2}(u)$ and $\theta(u, t)$ given by (9) and (10) has constant mean curvature $H$ if and only if $U(u)$ is given by:

1. if $\kappa=\tau=H=0$, 


$$
U^{2}(u)=\frac{u^{2}+a^{2}+c^{2} / 4}{m^{2}} ;
$$

2. if $\kappa=4 \tau^{2} \neq-H^{2}$,

$$
U^{2}(u)=\frac{c_{1}+\sqrt{c_{1}^{2}+c_{2}\left(H^{2}+4 \tau^{2}\right)} \sin \left(\sqrt{4 \tau^{2}+H^{2}} u\right)}{m^{2}\left(H^{2}+4 \tau^{2}\right)} ;
$$

3. if $-H^{2}=\kappa \neq 4 \tau^{2}$,

$$
U^{2}(u)=\frac{\left(\frac{b_{1}}{2} u^{2}+b_{2}\right)^{2}+b_{3}}{m^{2}\left(4 \tau^{2}+H^{2}\right)} ;
$$

4. if $-H^{2}<\kappa \neq 4 \tau^{2}$,

$$
U^{2}(u)=\frac{\left(H^{2}+\kappa\right)^{2} b_{3}+\left[b_{1}+\sqrt{b_{1}^{2}+b\left(H^{2}+\kappa\right)} \sin \left(\sqrt{H^{2}+\kappa} u\right)\right]^{2}}{m^{2}\left(4 \tau^{2}-\kappa\right)\left(H^{2}+\kappa\right)^{2}}
$$

5. if $-H^{2}>\kappa \neq 4 \tau^{2}$,

$$
U^{2}(u)= \begin{cases}\frac{\left(H^{2}+\kappa\right)^{2} b_{3}+\left[b_{1}-\sqrt{-b_{1}^{2}-b\left(H^{2}+\kappa\right)} \sinh \left(\sqrt{-\left(H^{2}+\kappa\right)} u\right)\right]^{2}}{m^{2}\left(4 \tau^{2}-\kappa\right)\left(H^{2}+\kappa\right)^{2}}, & b_{1}^{2}+b\left(H^{2}+\kappa\right)<0, \\ \frac{\left(H^{2}+\kappa\right)^{2} b_{3}+\left[b_{1}-\sqrt{b_{1}^{2}+b\left(H^{2}+\kappa\right)} \cosh \left(\sqrt{-\left(H^{2}+\kappa\right)} u\right)\right.}{m^{2}\left(4 \tau^{2}-\kappa\right)\left(H^{2}+\kappa\right)^{2}}, & b_{1}^{2}+b\left(H^{2}+\kappa\right)>0,\end{cases}
$$

where $b, b_{i}, c_{i} \in \mathbb{R}$, are the constants given by

$$
\begin{aligned}
b & =(1-2 a \tau)\left[\kappa(1+2 a \tau)-8 \tau^{2}\right]-c^{2}, & b_{1} & =4 \tau^{2}-2 a \kappa \tau-c H, \\
b_{2} & =-\frac{b}{2 b_{1}}, & b_{3} & =4 a \tau-a^{2} \kappa-1, \\
c_{1} & =1+(1-2 a \tau)^{2}-c H, & c_{2} & =-c^{2}-4 a^{2}(1-a \tau) .
\end{aligned}
$$

These expressions define a 1-parameter family $\left\{U_{c}(u)\right\}$ of functions $U(u)$ such that the helicoidal surface $\psi_{\left[U_{c}, m, a\right]}(u, t)$ has constant mean curvature and equal to $H$.

Proof Using the transformation of coordinates given by

$$
\left\{\begin{array}{l}
x(u)=m U(u), \\
y(u)=\sqrt{\frac{\left(x^{2}(u)-a^{2}\right)\left[(1+\sqrt{\Delta(u)})^{2}-4 \tau^{2} x^{2}(u)\right]}{[1-2 a \tau+\sqrt{\Delta(u)}]^{2}}-\frac{x(u)^{2} x^{\prime}(u)^{2}}{\Delta(u)}},
\end{array}\right.
$$

where 


$$
\Delta(u)=(1-2 a \tau)^{2}+\left(4 \tau^{2}-\kappa\right)\left(x^{2}(u)-a^{2}\right),
$$

equation (28) becomes

$$
H y(u)=\frac{2}{B(u)}-1-\left(\frac{x(u) x^{\prime}(u)}{\sqrt{\Delta(u)}}\right)^{\prime},
$$

with

$$
B(u)=2 \frac{1-2 a \tau+\sqrt{\Delta(u)}}{(1+\sqrt{\Delta(u)})^{2}-4 \tau^{2} x^{2}(u)} .
$$

Therefore,

$$
y^{\prime}(u)=\frac{x(u) x^{\prime}(u)}{y(u) \sqrt{\Delta(u)}}\left[\frac{2}{B(u)}-1-\left(\frac{x(u) x^{\prime}(u)}{\sqrt{\Delta(u)}}\right)^{\prime}\right]=\frac{H x(u) x^{\prime}(u)}{\sqrt{\Delta(u)}}
$$

and thus,

$$
y(u)= \begin{cases}\frac{H x^{2}(u)+c}{2 \sqrt{\Delta(u)}}, & 4 \tau^{2}-\kappa=0, \\ \frac{H \sqrt{\Delta(u)}+c}{4 \tau^{2}-\kappa}, & 4 \tau^{2}-\kappa \neq 0,\end{cases}
$$

where $c$ is an arbitrary constant. Consequently, we have the following cases:

Case $4 \tau^{2}-\kappa=0$ From (32) and (34), if we suppose that $1-2 a \tau>0$, then we have

$$
\left(2 x(u) x^{\prime}(u)\right)^{2}=4\left[(1-2 a \tau)-\tau^{2}\left(x^{2}(u)-a^{2}\right)\right]\left(x^{2}(u)-a^{2}\right)-\left(H x^{2}(u)+c\right)^{2} .
$$

Putting $z=x^{2}$, the above expression is transformed into the following

$$
z^{\prime}(u)=\sqrt{-\left(H^{2}+4 \tau^{2}\right) z^{2}(u)+2 c_{1} z(u)+c_{2}},
$$

where

$$
c_{1}=1+(1-2 a \tau)^{2}-c H, \quad c_{2}=-c^{2}-4 a^{2}(1-a \tau) .
$$

Consequently, 
(i) If $H^{2}+4 \tau^{2}=0$, we have that the $\mathrm{BCV}$-space is $\mathbb{R}^{3}$ and $c_{1}=2, c_{2}=-c^{2}-4 a^{2}$. Thus, adjusting the origin of $u$, we get

$$
\sqrt{z+\frac{c_{2}}{4}}=u
$$

and since $z=m^{2} U^{2}$ we have

$$
U^{2}(u)=\frac{u^{2}+a^{2}+c^{2} / 4}{m^{2}} .
$$

We observe that when $c=0$, the helicoidal surface $\psi_{[U, m, a]}$ is a helicoid.

ii) If $H^{2}+4 \tau^{2} \neq 0$, by integrating (35) we have that

$$
u=\frac{1}{\sqrt{H^{2}+4 \tau^{2}}} \sin ^{-1}\left(\frac{\left(H^{2}+4 \tau^{2}\right) z-c_{1}}{\sqrt{c_{1}^{2}+c_{2}\left(H^{2}+4 \tau^{2}\right)}}\right),
$$

up to a constant. Thus, as $z=m^{2} U^{2}$, it follows that

$$
\begin{aligned}
U^{2}(u) & =\frac{c_{1}+\sqrt{c_{1}^{2}+c_{2}\left(H^{2}+4 \tau^{2}\right)} \sin \left(\sqrt{4 \tau^{2}+H^{2}} u\right)}{m^{2}\left(H^{2}+4 \tau^{2}\right)} \\
& =\frac{1}{m^{2}\left(H^{2}+4 \tau^{2}\right)}\left[1+(1-2 a \tau)^{2}-c H\right. \\
& \left.+2 \sqrt{(1-2 a \tau)^{2}-H(1-2 a \tau)\left(H a^{2}+c\right)-\tau^{2}\left(H a^{2}+c\right)^{2}} \sin \left(\sqrt{H^{2}+4 \tau^{2}} u\right)\right] .
\end{aligned}
$$

Case $4 \tau^{2}-\kappa \neq 0$ In this case, as

$$
\begin{aligned}
& x^{2}(u)=\frac{1+a^{2} \kappa-4 a \tau-\Delta(u)}{\kappa-4 \tau^{2}}, \\
& \frac{x(u) x^{\prime}(u)}{\sqrt{\Delta(u)}}=\frac{(\sqrt{\Delta(u)})^{\prime}}{4 \tau^{2}-\kappa},
\end{aligned}
$$

from (32) and (34) we get

$$
\begin{aligned}
& (H \sqrt{\Delta(u)}+c)^{2}=\left(4 \tau^{2}-\kappa\right)^{2} y^{2}(u) \\
& =(1-2 a \tau-\sqrt{\Delta(u)})\left(\kappa+\kappa \sqrt{\Delta(u)}+2 a \kappa \tau-8 \tau^{2}\right)-\left((\sqrt{\Delta(u)})^{\prime}\right)^{2} .
\end{aligned}
$$

Thus,

$$
(\sqrt{\Delta(u)})^{\prime}=\sqrt{-\left(H^{2}+\kappa\right) \Delta(u)+2 b_{1} \sqrt{\Delta(u)}+b},
$$


where

$$
b=(1-2 a \tau)\left(\kappa(1+2 a \tau)-8 \tau^{2}\right)-c^{2}, \quad b_{1}=4 \tau^{2}-2 a \kappa \tau-c H .
$$

In the sequel, we integrate equation (38), up to a change of the origin of $u$, considering the following possibilities:

i) If $H^{2}+\kappa=0$, then we obtain that

$$
\sqrt{\Delta(u)}=\frac{b_{1}}{2} u^{2}+b_{2}
$$

with

$$
b_{1}=2 a \tau H^{2}+4 \tau^{2}-c H, \quad b_{2}=-\frac{b}{2 b_{1}} .
$$

Then, substituting in the first equation of (29), we get

$$
\begin{aligned}
U^{2}(u) & =\frac{1}{m^{2}}\left(\frac{\Delta(u)-(1-2 a \tau)^{2}}{4 \tau^{2}+H^{2}}+a^{2}\right) \\
& =\frac{\left(\frac{b_{1}}{2} u^{2}+b_{2}\right)^{2}+a^{2} H^{2}+4 a \tau-1}{m^{2}\left(4 \tau^{2}+H^{2}\right)} .
\end{aligned}
$$

ii) If $H^{2}+\kappa>0$, then the integration of (38) gives

$$
\sqrt{\Delta(u)}=\frac{1}{H^{2}+\kappa}\left[b_{1}+\sqrt{b_{1}^{2}+b\left(H^{2}+\kappa\right)} \sin \left(\sqrt{H^{2}+\kappa} u\right)\right] .
$$

Therefore, substituting in the first equation of (29), we obtain

$$
U^{2}(u)=\frac{\left(H^{2}+\kappa\right)^{2}\left(4 a \tau-a^{2} \kappa-1\right)+\left[b_{1}+\sqrt{b_{1}^{2}+b\left(H^{2}+\kappa\right)} \sin \left(\sqrt{H^{2}+\kappa} u\right)\right]^{2}}{m^{2}\left(4 \tau^{2}-\kappa\right)\left(H^{2}+\kappa\right)^{2}} .
$$

iii) If $H^{2}+\kappa<0$, then the integration of (38) gives

$$
\sqrt{\Delta(u)}= \begin{cases}\frac{1}{H^{2}+\kappa}\left[b_{1}-\sqrt{-b_{1}^{2}-b\left(H^{2}+\kappa\right)} \sinh \left(\sqrt{-\left(H^{2}+\kappa\right)} u\right)\right], & b_{1}^{2}+b\left(H^{2}+\kappa\right)<0, \\ \frac{1}{H^{2}+\kappa}\left[b_{1}-\sqrt{b_{1}^{2}+b\left(H^{2}+\kappa\right)} \cosh \left(\sqrt{-\left(H^{2}+\kappa\right)} u\right)\right], & b_{1}^{2}+b\left(H^{2}+\kappa\right)>0 .\end{cases}
$$

Therefore, substituting in the first equation of (29), we obtain

$$
U^{2}(u)=\left\{\begin{array}{lr}
\frac{\left(H^{2}+\kappa\right)^{2} b_{3}+\left[b_{1}-\sqrt{-b_{1}^{2}-b\left(H^{2}+\kappa\right)} \sinh \left(\sqrt{-\left(H^{2}+\kappa\right)} u\right)\right]^{2}}{m^{2}\left(4 \tau^{2}-\kappa\right)\left(H^{2}+\kappa\right)^{2}}, & b_{1}^{2}+b\left(H^{2}+\kappa\right)<0, \\
\frac{\left(H^{2}+\kappa\right)^{2} b_{3}+\left[b_{1}-\sqrt{b_{1}^{2}+b\left(H^{2}+\kappa\right)} \cosh \left(\sqrt{-\left(H^{2}+\kappa\right)} u\right)\right.}{m^{2}\left(4 \tau^{2}-\kappa\right)\left(H^{2}+\kappa\right)^{2}}, & b_{1}^{2}+b\left(H^{2}+\kappa\right)>0 .
\end{array}\right.
$$


Remark 3 In particular, if we consider $m=1$ and $a=a_{0}$ in the expressions of $U(u)$ obtained in Theorem 4, we see that an arbitrary helicoidal surface in $\mathcal{N}_{\kappa, \tau}$ has constant mean curvature $H$ if and only if the functions $\xi_{1}(u), \xi_{2}(u)$ and $\theta(u, t)$ are given by (9) and (10) by substituting the corresponding function $U(u)$.

Remark 4 Putting $\kappa=\tau=0$ in the equations (36) and (37), we obtain the following expressions:

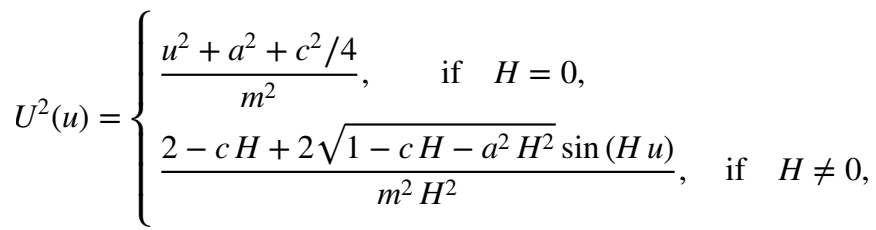

the second of which was given by Do Carmo and Dajczer in [18].

Example 2 (General helicoidal minimal surface in $\mathbb{R}^{3}$ ) Considering in $\mathbb{R}^{3}$ the function $U(u)=\sqrt{u^{2}+a^{2}+c^{2}}$, we obtain that the natural parametrization of a general helicoidal minimal surface given by

$$
\left\{\begin{array}{l}
\xi_{1}(u)=\sqrt{u^{2}+c^{2}}, \\
\xi_{2}(u)=c \cosh ^{-1}\left(\sqrt{\frac{u^{2}}{a^{2}+c^{2}}+1}\right)+a \arctan \left(\frac{a u}{c \sqrt{u^{2}+a^{2}+c^{2}}}\right), \\
\theta(u, t)=t-\arctan \left(\frac{a u}{c \sqrt{u^{2}+a^{2}+c^{2}}}\right) .
\end{array}\right.
$$

This family of surfaces called second Scherk's surfaces ${ }^{2}$ includes the catenoid and the helicoid that correspond to the cases $a=0$ and $c=0$, respectively. We observe that all the surfaces of the family for which the sum $a^{2}+c^{2}$ is the same are isometric to each other and, also, every helicoidal minimal surface in $\mathbb{R}^{3}$ belongs to one of the families of isometric surfaces obtained deforming catenoids into helicoids.

Remark 5 From Theorem 4, in the Heisenberg space $\mathbb{H}_{3}(\tau \neq 0$ and $\kappa=0)$ the helicoidal minimal surfaces are determined by the function

$$
U^{2}(u)=\frac{\left(2 \tau^{2} u^{2}+1-2 a \tau+\frac{c^{2}}{8 \tau^{2}}\right)^{2}+4 a \tau-1}{4 m^{2} \tau^{2}} .
$$

Therefore, among the helicoidal surfaces in $\mathbb{H}_{3}$ obtained in Example 1 the only minimal surface is the helicoidal catenoid because the function $U(u)=\left(u^{2}+2\right) / 2$ can be obtained just choosing in (43) $c^{2}=1=m$ and $a=1 / 2$.

\footnotetext{
${ }^{2}$ In 1835, H.F. Scherk made an important contribution to minimal surfaces theory with his work [37] that contains the first examples of minimal surfaces obtained from the integral of Monge and Legendre. Also, he investigated minimality of surfaces given as graphs $z=z(r, \theta)$ (where $(r, \theta, z)$ are cylindrical coordinates in $\mathbb{R}^{3}$ ) satisfying the condition $\frac{\partial^{2} z}{\partial r \partial \theta}=0$ and determined all the helicoidal minimal surfaces. Detailed accounts and further information can be found in [13], on p. 60, and in [15], on p. 327.
} 
Funding Open access funding provided by Università degli Studi di Cagliari within the CRUI-CARE Agreement.

Data Availibility Statement No datasets were generated or analyzed for the results of this paper.

Open Access This article is licensed under a Creative Commons Attribution 4.0 International License, which permits use, sharing, adaptation, distribution and reproduction in any medium or format, as long as you give appropriate credit to the original author(s) and the source, provide a link to the Creative Commons licence, and indicate if changes were made. The images or other third party material in this article are included in the article's Creative Commons licence, unless indicated otherwise in a credit line to the material. If material is not included in the article's Creative Commons licence and your intended use is not permitted by statutory regulation or exceeds the permitted use, you will need to obtain permission directly from the copyright holder. To view a copy of this licence, visit http://creativecommons.org/licenses/by/4.0/.

\section{References}

1. Alexandrino, M.M., Bettiol, R.G.: Lie groups and geometric aspects of isometric actions. Springer, Cham (2015)

2. Back, A., do Carmo, M.P., Hsiang, W.Y.: On some fundamental equations of equivariant Riemannian geometry. Tamkang J. Math. 40(4), 343-376 (2009)

3. Beltrami, E.: Risoluzione di un problema relativo alla teoria Delle Superfici gobbe. Ann. Mat. Pura Appl. 7, 139-150 (1865)

4. Bianchi, L.: Sugli spazi a tre dimensioni che ammettono un gruppo continuo di movimenti, Mem. Soc. It. delle Scienze (dei XL) (b), 11: 267-352 (1897)

5. Bianchi, L.: Gruppi continui e finiti. Ed. Zanichelli, Bologna (1928)

6. Bobenko, A.I., Eitner, U.: Bonnet surfaces and Painleve equations. J. Reine Angew. Math. 499, 47-79 (1998)

7. Bobenko, A.I., Eitner, U.: Painleve equations in the differential geometry of surfaces Lecture Notes in Mathematics, vol. 1753. Springer, Berlin (2000)

8. Bonnet, O.: Mémoire sur la théorie des surfaces applicables. J. Éc. Polyt 42, 72-92 (1867)

9. Bour, E.: Memoire sur le deformation de surfaces, pp. 1-148. XXXIX Cahier, J. Éc. Polyt. (1862)

10. Caddeo, R., Piu, P., Ratto, A.: $S O(2)$-invariant minimal and constant mean curvature surfaces in three dimensional homogeneous spaces. Manuscripta Math. 87, 1-12 (1995)

11. Cartan, É.: Leçons sur la géométrie des espaces de Riemann. Gauthier Villars, Paris (1946)

12. Cartan, É.: Sur les couples de surfaces applicables avec conservation des courbures principales. Bull. Sci. Math., (2) 66 (1942), 55-72, 74-85

13. Clapier, F.C.: Sur les surfaces minima ou élassoides. Thèses de l'entre-deux-guerres (1919)

14. Dajczer, M., Tenenblat, K.: Rigidity for complete Weingarten hypersurfaces. Trans. Amer. Math. Soc. 312(1), 129-140 (1989)

15. Darboux, G.: Leçons Sur la Théorie Générale des Surfaces. Vol. I, Paris (1914)

16. Delaunay, C.: Sur la surface de révolution dont la courbure moyenne est constante. J. Math. Pures et Appl. 1, 309-320 (1841)

17. Dini, U.: Sulle Superfici gobbe nelle quali uno dei due raggi di curvatura principale è una funzione dell'altro. Ann. Mat. Pura Appl. 7, 205-210 (1865)

18. do Carmo, M.P., Dajczer, M.: Helicoidal surfaces with constant mean curvature. Tôhoku Math. J. 34, 425-435 (1982)

19. Figueroa, C.B., Mercuri, F., Pedrosa, R.H.L.: Invariant surfaces of the Heisenberg groups. Ann. Mat. Pura Appl. 177, 173-194 (1999)

20. Fubini, G.: Sugli spazi che ammettono un gruppo continuo di movimenti, Ann. di Matem. Tomo 8, serie III, (1903), 39-82

21. Kenmotsu, K.: Surfaces of revolution with prescribed mean curvature. Tôhoku Math. J. 32, 147153 (1980)

22. Kühnel, W.: Ruled W-surfaces. Arch. Math. 62, 465-480 (1994)

23. Jensen, G.R., Musso, E., Nicolodi, L.: Compact Surfaces with no Bonnet Mate. J. Geom. Anal. 28(3), 2644-2652 (2018)

24. Minding, F.A.: Wie sich unterscheiden läßt, ob zwei gegebene krumme Fächen aufeinander abwickelbar sind oder nicht; nebst Bemerkungen über Fächen von unveränderlichem Krümmungsmaß. J. Reine Angew. Math. ( Giornale di Crelle) 19, 370-387 (1839) 
25. Montaldo, S., Onnis, I.I.: Invariant CMC surfaces in $\mathbb{\boxplus}^{2} \times \mathbb{R}$. Glasg. Math. J. 46, 311-321 (2004)

26. Montaldo, S., Onnis, I.I.: Invariant surfaces in a three-manifold with constant Gaussian curvature. J. Geom. Phys. 55, 440-449 (2005)

27. Montaldo, S., Onnis, I.I.: Invariant surfaces in $\mathbb{H}^{2} \times \mathbb{R}$ with constant (Gauss or mean) curvature. Publ. de la RSME 9, 91-103 (2005)

28. Montaldo, S., Onnis, I.I.: Geodesics on an invariant surface. J. Geom. Phys. 61, 1385-1395 (2011)

29. Onnis, I.I.: Invariant surfaces with constant mean curvature in $\mathbb{H}^{2} \times \mathbb{R}$. Ann. Mat. Pura Appl. 187, 667-682 (2008)

30. Piu, P.: Sur les flots riemanniens des espaces de D’Atri de dimension 3. Rend. Sem. Mat. Univ. Politec. Torino 46, 171-187 (1988)

31. Piu, P., Profir, M.M.: On the three-dimensional homogeneous SO(2)-isotropic Riemannian manifolds. An. Stiint. Univ. Al. I. Cuza Iasi. Mat. (N.S.) 57, 361-376 (2011)

32. Ripoll, J.B.: Superfícies invariantes de curvatura média constante em $Q^{3}$. Tese de Doutorado, IMPA (1986)

33. Ripoll, J.B.: Helicoidal minimal surfaces in hyperbolic space. Nagoya Math. J. 114, 65-75 (1989)

34. Roussos, I.M.: Principal curvature preserving isometries of surfaces in ordinary space. Bol. Soc. Brasil. Mat. 18(2), 95-105 (1987)

35. Roussos, I.M.: A geometric characterization of helicoidal surfaces of constant mean curvature. Publ. Inst. Math. (Beograd) (N.S.) 43(57), 137-142 (1988)

36. Sá Earp, R., Toubiana, E.: Screw motion surfaces in $\mathbb{\boxplus}^{2} \times \mathbb{R}$ and $\mathbb{S}^{2} \times \mathbb{R}$, Illinois. J. Math. 49, 1323$1362(2005)$

37. Scherk, H.F.: Bemerkungen uber die kleinste Fläche innerhalb gegebener Grenzen. J. für die reine und angewandte Mathematik 13, 185-208 (1835)

38. Scott, P.: The geometries of 3-manifolds. Bull. Lond. Math. Soc. 15, 401-487 (1983)

39. Vranceanu, G.: Leçons de géométrie différentielle. Ed. Acad. Rep. Pop. Roum., vol. I, Bucarest (1957)

40. Wunderlich, W.: Beitrag zur Kentnis der Minimalscharaubflachen. Compositio Math. 10, 297-311 (1952)

Publisher's Note Springer Nature remains neutral with regard to jurisdictional claims in published maps and institutional affiliations. 